\title{
Reading a series of similar texts: Testing a schema-based learning theory
}

\author{
MARK A. MCDANIEL \\ Purdue University, West Lafayette, Indiana \\ and \\ GILLES O. EINSTEIN \\ Furman University, Greenville, South Carolina
}

\begin{abstract}
Thorndyke and Hayes-Roth (1979) proposed, and presented data to support, a schema model of learning from simple texts. They assumed that repeated exposures to similar texts promoted acquisition of a schema for these texts, which in turn benefited recall for new instances of texts that conform to this schema. They further suggested that with repeated use of the schema, the increased number of details associated with the schema disrupted recall. Their data confirmed these expectations. However, Thorndyke and Hayes-Roth did not use a true 0-exposure control to assess baseline recall of the target text. We assessed baseline recall using both a true 0 -exposure control and Thorndyke and Hayes-Roth's derived 0 -exposure measure. The predicted inverted-U. shaped curve for recall as a function of prior training texts was replicated with Thorndyke and Hayes-Roth's baseline measure but not with a true 0 -exposure baseline. With the 0 -exposure baseline, recall was a monotonically decreasing function of number of training texts. This result suggests that recall of similar passages reflects nothing more or less prosaic than proactive interference.
\end{abstract}

During the last 15 years, the construct of higher order memory structures, called schemata, has been prominent in memory and cognition, particularly in the proseprocessing literature. In general, schemata are defined as knowledge clusters or structures that influence the degree to which incoming information is organized, the production of inferences, and retrieval processes (Pichert \& Anderson, 1977; Schank \& Abelson, 1977; van Dijk \& Kintsch, 1983; Yekovitch \& Thorndyke, 1981). One problem with schema theory, however, is that in some of its forms it is vague enough to be all encompassing and loses testability and empirical precision (Hintzman, in press; Thorndyke \& Hayes-Roth, 1979, p. 84).

One elegant application of the schema construct that has produced testable predictions, however, is that of Thorndyke and Hayes-Roth's (1979) model of learning from simple text. Thorndyke and Hayes-Roth proposed that repeated exposures to simple texts that shared some of the same semantic and structural features but differed in terms of particular details presented should promote the acquisition of a schema that included the common relations across the texts. For example, all texts might de-

Both authors contributed equally to this project; accordingly, order of authorship was determined arbitrarily. This research was supported by National Institute of Child Health and Human Development Grant HD23984. We are grateful to Perry Thorndyke for providing us with the texts. We also thank Mary-Michael Smeloff for testing subjects and Lindsay Holland for scoring the data. Correspondence concerning this article should be addressed to Mark A. McDaniel, Department of Psychology, Purdue University, 1364 Psychology Bldg., Room 3156, West Lafayette, IN 47907-1364. scribe facts about constellations, and these facts would be presented using identical predicates (e.g., "It was originally charted at...') but different details. The idea is that after several exposures to different instantiations of this text, learners would develop a schema for these texts that would contain the general concepts that were repeated. The schema would then be available to help encode and retrieve subsequent presentations of the same kind of text. After several more presentations, however, there would be a cost to using the schema. The increased number of details associated with the schema would compromise learners' abilities to correctly discriminate the context (text) in which the details occurred. This model predicts an inverted-U-shaped function for recall of a target text. Free recall should increase from a baseline condition with no previous exposure to the text to a condition with moderate exposure to training texts (e.g., two exposures) that are similar to the target text. Then free recall should decline with more extensive prior exposure (e.g., four) to the training texts.

Although Thorndyke and Hayes-Roth (1979) found a pattern consistent with this prediction, their study incorporated a methodological feature that may have biased the obtained pattern. There was no true baseline condition in which subjects studied and attempted to recall the target text in the absence of prior exposure to training texts. Instead, baseline free-recall levels were estimated by averaging over the recall of all facts from the target text that were unrelated to facts in the training texts. Thorndyke and Hayes-Roth did randomize the particular facts in the target text assigned to the unrelated condition and assigned 
to the condition in which the predicates were repeated but the details changed (labeled the changed condition). Presumably, then, there should not be item-selection artifacts in using the recall of unrelated items as an estimate of baseline recall. It is the case, however, that for all of the target texts in the Thorndyke and Hayes-Roth study, subjects were exposed to the theme in prior training texts. Thus, although the unrelated facts did not share a previously seen predicate, they still were related to a previously seen theme. Given this global thematic relationship between the unrelated facts and previous texts, one still might be unsure about the integrity of the baseline measure.

A more unambiguous test of the predictions derived from the schema-based learning model would be to use a baseline in which free recall of an entire target text, presented without related prior training passages, was measured. One major feature of the present experiment was the inclusion of this baseline condition. The other major feature was the addition of an encoding condition designed to offset the diminished discriminability of details associated with extensive schema use (i.e., after repeated exposure to training texts). In this encoding condition, all target texts were presented with some of the letters deleted from the words. This manipulation appears to increase proposition-specific processing, which, among other things, would include increased processing of the individual concepts (lexical items) that constitute the proposition (cf. McDaniel \& Kerwin, 1987). We anticipated that to the extent that schema learning was evident in this experiment, inducing a more distinctive encoding of the concepts of each proposition in the target text might parlay the usual cost of extensive schema use into increasing benefits (cf. Einstein, McDaniel, Owen, \& Cote, 1990).

\section{METHOD}

\section{Design and Subjects}

The design was a $2 \times 3$ mixed factorial with type of processing task (control, letter deletion) manipulated between subjects and number of training passages $(0,2$, or 4$)$ manipulated within subjects. The 12 subjects in each of the two groups were Furman University introductory psychology students who received course credit for participating.

\section{Materials and Procedure}

The materials were 1812 -sentence passages that were used in the Thorndyke and Hayes-Roth (1979) experiments. These included six passages from each of the three conceptual categories of constellations, islands, and diseases. The topic of each text from a particular conceptual category was about a different instance of the category (e.g., the constellations Pavo, Lepus, Eranus, Apus, Aquita, and Corvus). Four of the passages were training passages; the remaining two texts were target texts. The 12 sentences in each training passage had a corresponding sentence (same predicate, different detail) in each of the other training passages. Using the terminology of Thorndyke and Hayes-Roth, 6 of the target-text sentences were changed sentences; that is, they shared predicates with sentences in each of the four training passages. For example, 1 target sentence was "The constellation Pavo can be seen best in July," and this corresponded to the training sentence "The constellation Eranus can be seen best in November." The 6 other sentences in the target text were unrelated to sentences that had appeared in the training passages. (These were the sentences that Thorndyke and Hayes-
Roth used to establish their 0-baseline measure.) For example, the target sentence "This constellation can usually be seen after sunset" is unrelated to the training sentence "This constellation is commonly called 'the river." "The changed and unrelated sentences were different for the two target passages within each topic (the two target passages were counterbalanced across subjects as each subject received only one target passage per topic). For one target passage, the changed sentences were the odd-numbered sentences, and for the other, the changed sentences were the even-numbered sentences.

For the training phase, all subjects received six training texts-none from one category, two from a second category, and four from a third category. The order of text presentation was determined randomly for each subject, and the topics assigned to each training condition $(0,2$, or 4 training texts) were counterbalanced across subjects. Subjects in both the control and letter-deletion conditions received the same passages and the same processing instructions during the training phase. Specifically, they were told to read the passages at their own pace, and to read them carefully because they would later receive a memory test. The passages were presented to the subjects in a booklet, with one passage on each page, and the subjects were not allowed to turn back to a previous passage. After the subjects completed reading the last text, they were given a 2-min math distractor task.

The subjects then proceeded to the test phase of the experiment, in which they received three target passages in a randomly determined order (one from each of the three conceptual categories of constellations, islands, and diseases). Also, one target passage corresponded to each of the training conditions $(0,2$, or 4 training texts). Across subjects, each of the passages for each topic was used equally often. Subjects in the control condition received intact passages and the same intentional learning instructions as those used in the training phase. Subjects in the letterdeletion condition received passages in which $33 \%$ of the letters were deleted (approximately $40 \%$ of the deleted letters were vowels) and replaced by blanks. The exact locations of the deletions were determined haphazardly, and pilot research was used to ensure that most subjects could successfully fill in the correct letters. Letter-deletion subjects were told to fill in as many missing letters as possible while reading the passage. Moreover, like control subjects, they were told to read the passage carefully because they would later receive a memory test.

After processing their respective texts, all subjects were given a 2min math distractor task, followed by a free-recall test for the three target passages. The subjects were asked to recall the passages in the order in which the passages had been presented, and they were given as much time as needed.

\section{RESULTS}

The alpha level was set at .05 for all analyses. The basic analysis was a $2 \times 3$ mixed analysis of variance (ANOVA) with encoding condition (intact text, letterdeleted text) as the between-subjects variable and number of prior training texts $(0,2,4)$ as the within-subjects variable. To ensure that the Thorndyke and Hayes-Roth (1979) results were replicated, we first analyzed the data using the baseline free recall ( 0 prior exposure) used by Thorndyke and Hayes-Roth. That is, we averaged over the recall of all unrelated facts from the target texts for which there were 2 or 4 prior training texts, and used this as an estimate of the number of changed facts recalled for the 0-prior-exposure cell. For the 2 and 4 priortraining-text cells, the recall of changed facts was scored directly (for the detail recall measure, a verbatim criterion was used; otherwise a gist scoring criterion was used). An ANOVA was computed for each of three dependent measures: recall of entire sentences, recall of predicates, and recall of details (refer to Table 1 for means). ${ }^{1}$ For all measures, there was a significant effect 
Table 1

Proportion Free Recall of Changed Sentences and of the Sentence Constituents (Predicates and Details)

\begin{tabular}{|c|c|c|c|c|c|c|c|c|c|}
\hline \multirow{3}{*}{$\begin{array}{c}\text { Number of } \\
\text { Training } \\
\text { Passages }\end{array}$} & \multicolumn{9}{|c|}{ Encoding Task } \\
\hline & \multicolumn{3}{|c|}{ Sentence Recall } & \multicolumn{3}{|c|}{ Predicate Recall } & \multicolumn{3}{|c|}{ Detail Recall } \\
\hline & $\begin{array}{c}\text { Intact } \\
\text { Text }\end{array}$ & $\begin{array}{l}\text { Letters } \\
\text { Deleted }\end{array}$ & Mean & $\begin{array}{c}\text { Intact } \\
\text { Text }\end{array}$ & $\begin{array}{l}\text { Letters } \\
\text { Deleted }\end{array}$ & Mean & $\begin{array}{c}\text { Intact } \\
\text { Text }\end{array}$ & $\begin{array}{l}\text { Letters } \\
\text { Deleted }\end{array}$ & Mean \\
\hline Estimated $0 *$ & .30 & .13 & .22 & .30 & .15 & .22 & .15 & .07 & .11 \\
\hline 0 & .51 & .31 & .41 & .53 & .33 & .43 & .39 & .21 & .30 \\
\hline 2 & .46 & .26 & .36 & .46 & .31 & .38 & .28 & .18 & .23 \\
\hline 4 & .39 & .24 & .32 & .39 & .28 & .34 & .21 & .15 & .18 \\
\hline
\end{tabular}

*Estimated 0 is the average number of unrelated facts in the target texts from the 2 and 4 training conditions.

of number of training texts [for sentence recall, $F(2,44)$ $=3.98, M S_{\mathrm{e}}=1.20$; for predicate recall, $F(2,44)=$ $5.22, M S_{\mathrm{e}}=1.06$; and for detail recall, $F(2,44)=3.76$, $M S_{\mathrm{e}}=.86$ ]. Inspection of Table 1 reveals that the training-text effect reproduced the inverted-U-shape function reported by Thorndyke and Hayes-Roth. Subsequent planned comparisons indicated that the quadratic component was significant for sentence recall $[F(1,44)=4.64]$, predicate recall $[F(1,44)=5.61]$, and detail recall $[F(1,44)=4.84]$. There were no reliable linear components for sentence recall $(F=3.36)$ or detail recall $(F=$ 2.66), whereas there was a reliable linear component for predicate recall $[F(1,44)=4.64]$. This effect in predicate recall is similar to Thorndyke and Hayes-Roth's finding of monotonically increasing recall of predicates.

There was one other significant effect. Intact target texts were recalled significantly better than letter-deleted texts when recall for the entire sentence was considered $\left[F(1,22)=6.74, M S_{e}=2.82\right]$. With an average of $98.3 \%$ of the letters being filled in, this effect was clearly not due to an inability of subjects to fill in the missing letters.

The results changed dramatically, however, when the analyses were recomputed using the true 0 -exposure baseline (i.e., recall of changed facts from the text with no prior training texts). There were no significant effects of number of training texts. As can be seen from Table 1, the levels of recall for the changed facts were nominally higher for the 0-exposure baseline than for the " 2 "' and " 4 " conditions, creating a monotonically decreasing function. Indeed, a comparison performed on the means indicated a reliable linear component for detail recall $[F(1,44)$ $\left.=4.48, M S_{\mathrm{e}}=1.34\right]$. By contrast, there was no evidence of a quadratic trend for any of the dependent measures (largest $F<1$ ). These analyses also showed that intact texts were recalled significantly better than were letterdeleted texts (this effect did not interact with number of training texts) [for sentence recall, $F(1,22)=7.36, M S_{\mathrm{e}}$ $=3.02$; for predicate recall, $F(1,22)=4.35, M S_{\mathrm{e}}=$ 3.47; and for detail recall, $\left.F(1,22)=4.53, M S_{\mathrm{e}}=1.77\right]$.

In short, the values obtained using recall of unrelated facts to estimate recall levels of changed facts for the " 0 " condition were quite different from those obtained from the actual recall levels of changed facts in the " 0 " condition. Recall of changed facts in the " 0 " condition were significantly higher than the estimations based on recall of unrelated facts [for sentence recall, $F(1,22)=19.45$, $M S_{\mathrm{e}}=.84 ;$ for predicate recall, $F(1,22)=27.06, M S_{\mathrm{e}}$ $=.67$; and for detail recall, $\left.F(1,22)=16.75, M S_{\mathrm{e}}=.94\right]$. When the actual recall levels of changed facts are considered, what at first appeared to be a U-shaped function is more nearly a linear function, with recall decreasing as more and more details are introduced by increasing numbers of training texts.

\section{DISCUSSION}

At first glance, the inverted-U-shaped recall pattern reported by Thorndyke and Hayes-Roth (1979) seems to offer impressive support for a schema-based model of learning. The reliability of this pattern is not in question, as we were able to replicate it when using the baseline recall measure used by Thorndyke and Hayes-Roth. The current findings question the implications of that pattern, however. With a more straightforward and appropriate baseline (recall of changed sentences from a text with 0-prior-training passages), the quadratic component of the recall function disappeared. This change is due to the fact that the true 0 -level baseline for changed facts was significantly higher than the baseline calculated from the unrelated facts of the texts in the " 2 " and " 4 " conditions.

If anything, recall appears to be a decreasing monotonic function of number of training passages. This trend is inconsistent with the idea that a schema is acquired through experience with related training passages and subsequently instantiated for encoding and retrieval of the target passage. The negative effects of the letter-deletion variable converge on this conclusion. Previous work has shown that letter-deleted passages are better recalled than intact passages for texts for which subjects can instantiate a schema (e.g., fairy tales or an ambiguous passage for which the theme-e.g., "washing clothes"'-is supplied; Einstein, McDaniel, Bowers, \& Stevens, 1984). For texts for which it is unlikely that the reader would have acquired a schema (e.g., descriptive passages about avalanches in the Kanchenjunga Mountains), deleting letters does not benefit recall (McDaniel, Einstein, Dunay, \& Cobb, 1986). Thus, the present negative mnemonic effect of letter deletion implies that extensive schema learning did not occur in this paradigm.

In conclusion, it appears that the type of recall function one obtains will depend in part on the type of baseline used. It should be noted, however, that there are other methodological differences between our study and that of Thorndyke and Hayes-Roth (1979) that may have contributed to the different effects of number of training passages. ${ }^{2}$ Nevertheless, our data using a true 0-level baseline (i.e., recall of a target text with no prior related training passages) suggest that the mnemonic effects of training passages primarily reflect simple proactive interference.

\section{REFERENCES}

Einstein, G. O., McDaniel, M. A., Bowers, C. A., \& Stevens, D. T. (1984). Memory for prose: The influence of relational and propositionspecific processing. Journal of Experimental Psychology: Learning, Memory, \& Cognition, 10, 133-143. 
Einstein, G. O., McDaniel, M. A., Owen, P. D., \& Cote, N. C. (1990). Encoding and recall of texts: The importance of material appropriate processing. Journal of Memory \& Language, 29, 566-581. HintzMAN, D. (in press). 25 years of learning and memory: Was the cognitive revolution a mistake? In D. E. Meyer \& S. Kornblum (Eds.), Attention and performance XIV: $A$ silver jubilee. Hillsdale, NJ: Erlbaum.

McDaniel, M. A., Einstein, G. O., Dunay, P. K., \& Cobb, R. E. (1986). Encoding difficulty and memory: Toward a unifying theory. Journal of Memory \& Language, 25, 645-656.

MCDANIEL, M. A., \& KeRWIN, M. L. E. (1987). Long-term prose retention: Is an organizational schema sufficient? Discourse Processes, 10, 237-252.

Pichert, J. W., \& ANDerson, R. C. (1977). Taking a different perspective on a story. Journal of Educational Psychology, 69, 309-315.

SCHANK, R., \& ABELSON, R. (1977). Scripts, plans, goals, and understanding. Hillsdale, NJ: Erlbaum.

THORNDYKE, P. W., \& HAYES-RoTh, B. (1979). The use of schemata in the acquisition and transfer of knowledge. Cognitive Psychology, 11, 82-106.

VAN Dijk, T. A., \& Kintsch, W. (1983). Strategies of discourse comprehension. New York: Academic Press.

YeKovich, F. R., \& THORNDYKe, P. W. (1981). An evaluation of alternative functional models of narrative schemata. Journal of Verbal Learning \& Verbal Behavior, 20, 454-469.

\section{NOTES}

1. Note that the scores are tabled as proportions, but that the statistical analyses used number correct.

2. We used a total of 6 training passages, whereas Thorndyke and Hayes-Roth (1979) used 18. Also, in the Thorndyke and Hayes-Roth passages but not those in the present study, there were entire sentences repeated across training and target texts.

(Manuscript received December 22, 1990.) 